\title{
DETERMINAÇÃO DE ISOTIOCIANATO DE BENZILA EM Carica papaya UTILIZANDO CROMATOGRAFIA GASOSA COM DETECTORES SELETIVOS
}

\author{
Izabela Miranda de Castro* e Marianna Ramos dos Anjos \\ Laboratório de Resíduos de Agrotóxicos, Embrapa Agroindústria de Alimentos, Av. das Américas, 29501, $23020-470$ Rio de \\ Janeiro - RJ, Brasil \\ Elba dos Santos de Oliveira \\ Laboratório de Gases Combustíveis, Instituto Nacional de Tecnologia, Av. Venezuela, 82, 20081-312 Rio de Janeiro - RJ, Brasil
}

Recebido em 6/8/07; aceito em 3/7/08; publicado na web em 5/11/08

\begin{abstract}
DETERMINATION OF BENZYL ISOTHIOCYANATE IN Carica papaya USING GAS CHROMATOGRAPHY WITH SELECTIVES DETECTORS. In the present work, a method was developed and validated for the quantification of benzyl isothiocyanate (BITC) in the fruits of Carica papaya. The quantification of this compound was carried out by gas chromatography (GC) with selective detectors - nitrogen phosphorus detector (NPD) and flame photometric detector (FPD). The performance of these detectors showed a higher sensitivity of the NPD with a broader linear range of detection. The LOD/LOQ were $0.038 / 0.100 \mu \mathrm{g} / \mathrm{mL}$ for NPD and 5.78/19.29 $\mu \mathrm{g} / \mathrm{mL}$ for FPD. The recovery of the method for BITC was 90,64\%. An average value of BITC concentration in all the analyzed samples was $16,23 \mu \mathrm{g}$ BITC/g.
\end{abstract}

Keywords: isothiocyanates; Carica papaya; gas chromatography.

\section{INTRODUÇÃO}

Os glicosinolatos (ß-tioglicosídio-N-hidroxissulfatos) são precursores dos isotiocianatos, e ocorrem em 16 famílias de espécies vegetais, existindo, pelo menos, 120 estruturas diferentes identificadas em plantas. Uma grande parte das espécies que sintetizam estas substâncias pertence aos gêneros Brassicaceae, Caricaceae e Capparaceae. ${ }^{1}$

Os glicosinolatos são convertidos nas plantas em isotiocianatos por ação da enzima mirosinase. Esta enzima, presente no próprio vegetal ou na microflora do trato digestivo humano, inicia sua atividade quando o tecido vegetal sofre algum tipo de injúria ou sob ação de fungos ou, ainda, pela mastigação de insetos ou, então, pela própria mastigação das plantas frescas. ${ }^{2}$ Alguns estudos mencionam efeitos nocivos dos glicosinolatos na saúde: a formação de bócio, ${ }^{3}$ características antinutricionais e toxicidade causadas pela dieta, ${ }^{4}$ efeitos citotóxicos e a indução de aberrações cromossomiais constam da literatura. ${ }^{5-8}$

Existe, no entanto, uma dicotomia no que tange os efeitos de glicosinolatos e isotiocianatos na dieta diária. Vários estudos registram a ocorrência de glicosinolatos em vegetais que constituem uma parcela importante da dieta da população. Muitas evidências mostram que o aumento do consumo de frutas e vegetais reduz a incidência de câncer, em alguns casos, na ordem de 50\%. ${ }^{9}$ As propriedades químio-protetoras dos vegetais são atribuídas à ocorrência em baixas concentrações de certas substâncias como os glicosinolatos. Alguns destes induzem os mecanismos químicos de defesa contra agentes cancerígenos aumentando a resistência celular. Os glicosinolatos formam tiocianatos, isotiocianatos e nitrilas. ${ }^{1,10}$ Os isotiocianatos atuam tanto com os indutores das enzimas da Fase 2 do mecanismo de desintoxicação celular, ${ }^{11,12}$ mas também com o papel inibitório das enzimas da Fase 1, ${ }^{10,13,14}$ acentuando o desempenho celular no processo de defesa de desintoxicação química. ${ }^{1,15}$

O brócolis (brássica), importante fonte de glicosinolato e isotiocianatos, possui substâncias que são capazes de ativar a fase 2 de

*e-mail: imcastro@ctaa.embrapa.br desintoxicação celular e de proteger contra o câncer. ${ }^{16,17} \mathrm{O}$ isotiocianato de benzila, encontrado no mamão, pode agir como um inibidor no desenvolvimento de câncer de pâncreas ${ }^{18}$ e também como antihelmíntico. ${ }^{19}$ Os isotiocianatos de benzila e de feniletila exibiram atividade sobre o câncer de pulmão, aumentando a ocorrência de apoptosis das células cancerosas. ${ }^{20,21}$

De forma bem conclusiva, a atividade anti-carcinogênica dos isotiocianatos (ITCs) já foi comprovada e depende, em grande parte, do seu acúmulo no interior das células. ${ }^{22}$ Os isotiocianatos estimulam os mecanismos químio-protetores, mas, dependendo da sua concentração, podem também induzir o estresse celular. As doses de ITCs abaixo de certo nível acentuam o mecanismo de defesa. Por outro lado, as concentrações de ITCs, acima de um limite, favorecem o estresse celular e seus efeitos tóxicos. ${ }^{23} \mathrm{O}$ BITC induz a apoptosis das células de câncer de pulmão em baixas concentrações $(<10 \mu \mathrm{M})$, mas causa até a necrose do tecido em concentrações de $25 \mu \mathrm{M} .^{21}$

O consumo de ITCs, disponíveis ao ser humano através da dieta, mostra uma relação inversa ao risco de câncer. Estes resultados sugerem seu uso como um tratamento preventivo de câncer ou como agente terapêutico. ${ }^{24} \mathrm{O}$ mamão é um fruto amplamente consumido pela população brasileira e, por conseguinte, constitui-se em um item importante da dieta. A presença de isotiocianato de benzila no mamão (Carica papaya) já foi relatada em diferentes ocasiões. ${ }^{1,14,19,25}$ Neste fruto foi constatado que a concentração de BITC aumenta nas sementes e diminui na polpa, à medida que o fruto amadurece. Da mesma forma, no fruto verde, a concentração de glicosinolato é maior que após o amadurecimento. ${ }^{1}$ No que tange à segurança alimentar e o uso de propriedades funcionais dos alimentos, torna-se muito importante conhecer-se a qualidade do alimento que se está ingerindo e os seus efeitos na saúde humana após a ingesta. Assim, este estudo teve por objetivo estabelecer e validar um método de análise para determinar as concentrações de isotiocianatos presentes em alimentos, mais especificamente em mamão (Carica papaya). A aplicação deste método de análise de isotiocianatos nas amostras de mamão permitiu uma avaliação preliminar da sua concentração e sua distribuição no produto. 


\section{PARTE EXPERIMENTAL}

\section{Amostras}

Foram utilizadas amostras de mamão verde, predominantemente com grau de maturação 2, 3 ou 4, ou seja, frutos com 10-25\%, 25-40\% e $40-55 \%$ da área superficial da casca na cor amarela, segundo uma escala de maturação elaborada por Balbino. ${ }^{26}$ As amostras provenientes de cultivo convencional foram adquiridas no mercado local; as amostras cultivadas de forma orgânica foram coletadas no campo experimental da Embrapa Agrobiologia. Cada fruto foi considerado como uma amostra e cada amostra foi analisada em duplicata.

\section{Preparo das amostras}

Durante esta etapa, todas as amostras foram cuidadosamente manuseadas no laboratório usando-se luvas descartáveis para evitar contaminações e danos ou injúrias ao fruto. Depois de retiradas as sementes, a polpa e a casca foram cortadas, utilizando facas de aço inox, em pequenos cubos de $0,5 \mathrm{~cm}$, imediatamente antes da extração.

\section{Extração}

Efetuou-se a extração de cerca de $20 \mathrm{~g}$ de amostra (polpa+casca) com acetato de etila, durante $15 \mathrm{~min}$ em um homogeneizador de alta rotação (Ultra-Turrax). Após filtração em papel de filtro rápido, a solução foi evaporada em rota-evaporador e transferida para balão volumétrico de 10 ou $25 \mathrm{~mL}$ com acetato de etila. A solução contendo o analito foi aplicada em um sistema cromatográfico composto por um cromatógrafo gasoso com um detector de fotometria de chama (sistema CG-DFC) ou com detector de nitrogênio fósforo (sistema CG-DNP).

\section{Padrão de isotiocianato de benzila}

Utilizou-se isotiocianato de benzila 99,7\% (BITC) fabricado pela Fluka, referência 13505.

\section{Soluções padrão de BITC}

As soluções padrão foram feitas utilizando o padrão BITC da Fluka em acetato de etila. Foram preparadas soluções de BITC nas concentrações de 5,18; 10,36; 16,86; 22,48; 33,72; 44,96 e 56,20 $\mu \mathrm{g}$ / $\mathrm{mL}$ para serem usadas no sistema CG-DFC; soluções de BITC com 0,$10 ; 5,02 ; 10,03 ; 20,06 ; 30,09 ; 40,12$ e $50,15 \mu \mathrm{g} / \mathrm{mL}$ foram usadas no sistema CG-DNP.

\section{Sistemas e parâmetros cromatográficos}

Utilizou-se um cromatógrafo gasoso Trace 2000, fabricado pela Thermo $^{\circledR}$, equipado com um detector de fotometria de chama com filtro de enxofre (sistema CG-DFC) ou com um detector de nitrogênio fósforo (sistema CG-DNP); coluna DB-1701 P (28,5 m x 0,32 mm x $0,25 \mu \mathrm{m}$ ) fabricada pela $\mathrm{J} \& \mathrm{~W}$ Scientific; programação de temperatura, 50 a $130{ }^{\circ} \mathrm{C}\left(25^{\circ} \mathrm{C} / \mathrm{min}\right)$ e de 130 a $200{ }^{\circ} \mathrm{C}\left(5^{\circ} \mathrm{C} / \mathrm{min}\right)$; injetor splitless a $225^{\circ} \mathrm{C}$ (tempo de abertura da válvula de $1 \mathrm{~min}$ ); o volume injetado corresponde ao volume da agulha de $10 \mu \mathrm{L}$ Hamilton $701 \mathrm{~N}$ $(0,8 \mu \mathrm{L})$; fluxo de gás de arraste (hélio) de $1,1 \mathrm{~mL} / \mathrm{min}$. Detector DFC: temperatura da base $300{ }^{\circ} \mathrm{C}$; temperatura do detector $150{ }^{\circ} \mathrm{C}$; fluxo de hidrogênio de $90 \mathrm{~mL} / \mathrm{min}$; fluxo de ar sintético $105 \mathrm{~mL} / \mathrm{min}$. Detector DNP: temperatura da base $300{ }^{\circ} \mathrm{C}$; fluxo de hidrogênio de $2,3 \mathrm{~mL} / \mathrm{min}$; fluxo de ar sintético $60 \mathrm{~mL} / \mathrm{min}$; fluxo de nitrogênio (make up) $30 \mathrm{~mL} / \mathrm{min}$; corrente da pérola $2,35 \mathrm{pA}$.

\section{Análise por CG-DFC e CG-DNP}

Foram feitas análises em triplicata das soluções padrão de BITC e obtidas as curvas padrão de isotiocianato de benzila por cromatografia gasosa usando os detectores DFC e DNP. Os extratos das amostras de mamão foram analisados nos sistemas CG-DFC e CG-DNP em duplicata por padronização externa através da curva padrão.

\section{Validação da metodologia e avaliação de critérios de desempenho}

A validação de um procedimento analítico tem como objetivo a comprovação de que o método a ser utilizado é apropriado à finalidade para a qual foi desenvolvido. A determinação dos parâmetros de validação do método faz-se necessária para verificar o cumprimento de alguns critérios de desempenho. Neste estudo, foram aplicados os critérios/recomendações técnicas da Comunidade Européia. ${ }^{27,28}$ Os parâmetros considerados incluem a seletividade, faixa de trabalho, linearidade (coeficiente de correlação, homo/heterocedasticidade, F), taxa de recuperação e limites de quantificação e de detecção.

\section{Seletividade}

$\mathrm{O}$ isotiocianato de benzila, que é de ocorrência natural na matriz mamão, pode ser identificado sem interferência de outros componentes da matriz pelos detectores seletivos, DFC e DNP, usados nos dois sistemas cromatográficos. A injeção de uma amostra de mamão no sistema CG-DFC mostra apenas um pico com o mesmo tempo de retenção do padrão de BITC. Neste sistema cromatográfico foram analisadas amostras de mamão seguindo o protocolo experimental previamente descrito. A identificação do pico de interesse - BITC - nas amostras de mamão ocorreu através da comparação entre os tempos de retenção do padrão e os tempos de retenção dos picos observados nos cromatogramas das amostras. Como o detector DFC é seletivo e só responde às substâncias sulfuradas, obteve-se cromatogramas "limpos" sem a presença de interferentes. ${ }^{29}$ De forma análoga, utilizando o sistema cromatográfico com um detector seletivo de nitrogênio-fósforo (CG-DNP), a injeção de uma amostra de mamão gera um cromatograma com apenas um pico com o mesmo $t_{R}$ do padrão de BITC.

A confirmação destes resultados foi feita por espectrometria de massas (sistema CG-DSM). Neste caso foram utilizados os mesmos parâmetros analíticos usados nos sistemas com DFC e DNP (coluna, programação de temperatura do forno, fluxo de gás, injeção), confirmando a identidade do pico da amostra. Este pico tem o mesmo $t_{R}$ que o padrão de BITC e apresenta um espectro de massa compatível com o isotiocianato de benzila, segundo a comparação com espectros da biblioteca Wiley e do próprio padrão de BITC.

\section{Linearidade e faixa de trabalho}

A faixa de trabalho do método em estudo corresponde ao intervalo de concentrações do analito usadas na composição da curva de calibração que deve, obrigatoriamente, apresentar linearidade. A construção da curva analítica através do método dos mínimos quadrados permite a quantificação do analito caso a curva obedeça a certos critérios de desempenho.

A avaliação da homogeneidade na variância dos resíduos (homo/ heterocedasticidade) indica se em todo o intervalo de trabalho considerado o método dos mínimos quadrados pode ou não ser usado na análise de regressão da reta. Para isso aplica-se o teste de Cochran (C), onde se calcula o valor de $\mathrm{C}=\mathrm{s}_{\text {maxx }}{ }^{2} / \sum \mathrm{s}^{2}$ que é a relação entre a maior variância obtida e o somatório das variâncias, sendo que o valor de $\mathrm{C}_{\text {calculado }}$ deve ser inferior ao $\mathrm{C}_{\text {tabelado }}$.

Os coeficientes de correlação $\left(\mathrm{R}^{2}\right)$ e o de correlação linear (r), 
usados para avaliar a linearidade de curvas, revelam a relação entre concentração $\mathrm{x}$ área, mas não devem ser empregados isoladamente para esta finalidade. Os valores de $r$ pertencem ao intervalo $-1 \leq r \leq 1$, sendo que quanto mais próximo da unidade melhor a correlação entre $\mathrm{x}$ e $\mathrm{y}$, e os valores próximos de zero sugerem ausência de relação linear entre $\mathrm{x}$ e $\mathrm{y}$.

A avaliação de um modelo indicando a significância estatística da curva ajustada é feita pelo teste F. Para isso foi utilizada a ferramenta análise de variância da regressão (ANOVA) disponível no Excel (MS Office) para se confirmar a linearidade da curva. O teste de hipótese da significância da regressão, onde a hipótese nula $\left(\mathrm{H}_{0}\right)$ considera o coeficiente angular $b=0$ e na hipótese alternativa $\left(\mathrm{H}_{1}\right), \mathrm{b} \neq 0$. Se o valor de $\mathrm{F}_{\text {calculado }}>\mathrm{F}_{\text {de significação, }}$, hipótese nula é rejeitada e a alternativa aceita, evidenciando a linearidade no intervalo estudado.

$\mathrm{O}$ valor $\mathrm{P}$, probabilidade de hipótese nula, calculado através da ANOVA, deve ser maior ou igual a 0,05 em relação ao coeficiente linear, isto é, a reta deve passar próxima à origem. Caso contrário indica que o método está sujeito a erros sistemáticos.

\section{Ensaios de recuperação de BITC}

Primeiramente, a concentração original de isotiocianato de ocorrência natural (valor base) na amostra de mamão usada nestes ensaios foi determinada em 10 replicatas; nestas análises foram usados os procedimentos otimizados do modo de preparo e extração seguidos da quantificação pelo sistema CG-DNP. O mesmo valor base de BITC na mesma amostra foi calculado através de uma curva de adição de isoticianato à matriz também por CG-DNP. Os dois resultados obtidos (média das 10 replicatas e valor calculado por curva de adição) mostraram coerência entre si.

Para os ensaios de recuperação de BITC, realizados usando $25 \mathrm{~g}$ desta mesma amostra, foi aplicado exatamente o mesmo procedimento experimental usado para se determinar o valor base de isotiocianato; os ensaios foram feitos em triplicatas para três níveis de fortificação, 1,40; 2,80 e 4,15 $\mu \mathrm{g}$ de BITC/g de amostra. O valor base encontrado foi acrescido à quantidade teórica de BITC adicionada em cada nível de fortificação para efeito do cálculo da \% de recuperação. Todas as análises seguiram os procedimentos descritos anteriormente.

\section{Limites de detecção e quantificação}

Os limites de detecção (LD) e de quantificação (LQ) são as menores concentrações do analito que se pode detectar e quantificar, respectivamente, com precisão e exatidão. Quando se utilizou o sistema CG-DFC, o LD e o LQ foram calculados utilizando dados da regressão da curva de calibração calculada pela ferramenta Regressão disponível no software Excel (MS Office). O erro padrão da curva foi calculado e este valor usado para o cálculo dos limites de detecção e quantificação, sendo $\mathrm{LD}=3 \mathrm{~s} / \mathrm{b}$ e $\mathrm{LQ}=10 \mathrm{~s} / \mathrm{b}$, onde s é o desvio (erro padrão) e b é o coeficiente angular da reta.

Quando se utilizou o sistema CG-DNP, o cálculo dos limites de detecção e de quantificação foi feito utilizando a relação sinal/ruído, onde é aceita a razão 3:1 e 10:1 para o LD e LQ, respectivamente. Para se estabelecer estes limites foi usada a menor concentração de BITC capaz de fornecer um pico que tenha três ou dez vezes a amplitude do sinal de ruído. Os ensaios foram feitos em triplicata para cada concentração testada.

\section{RESULTADOS E DISCUSSÃO}

O modo de preparo e a extração das amostras são as etapas mais críticas deste método. A concentração de BITC nos frutos, que é gerado pela ação da mirosinase sobre os glicosinolatos após algum tipo de injúria, ${ }^{2}$ sofre a influência do tipo de processamento empregado.

Foram testados dois modos de preparo da amostra: homogenei- zada em um processador ou cortada em cubos. Usando-se acetato de etila como solvente, foram avaliadas duas temperaturas e diferentes formas de extração: agitação magnética, Ultra-Turrax (homogeneizador/dispersor de alta rotação), ultrassom ou um gral e, também, a adição de celite. Os resultados obtidos para a quantificação de BITC em mamão no sistema CG-DFC, utilizando os diferentes modos de preparo e extração, estão expostos na Tabela 1.

Tabela 1. Resultados da determinação de BITC por CG-DFC em mamão após diferentes tipos de preparo de amostra e modos de extração

\begin{tabular}{|c|c|c|c|c|c|c|c|}
\hline \multirow{4}{*}{ Modo de extração } & \multicolumn{7}{|c|}{ BITC ( $\mu \mathrm{g} / \mathrm{g}$ de amostra) } \\
\hline & \multicolumn{7}{|c|}{ amostras } \\
\hline & 1 & & 2 & & 3 & & 4 \\
\hline & \multicolumn{4}{|c|}{ AcOEt temp. amb. } & \multicolumn{3}{|c|}{ AcOEt a $4{ }^{\circ} \mathrm{C}$} \\
\hline \multicolumn{8}{|c|}{ preparo: cortada em cubos } \\
\hline Turrax & 19,2 & 19,6 & 12,5 & 14,1 & 8,2 & 8,1 & 11,1 \\
\hline US & 7,1 & 7,0 & & & & & \\
\hline Gral & 7,0 & & & & & & \\
\hline \multirow[t]{2}{*}{ Turrax c/ celite } & 8,0 & 7,6 & & & & & \\
\hline & \multicolumn{7}{|c|}{ preparo: homogeneizada em processador } \\
\hline Turrax & nd & nd & & & & & \\
\hline US & & & nd & nd & & & \\
\hline Agitação & & & & & nd & nd & \\
\hline
\end{tabular}

nd - não detectado. US - ultrassom

Os resultados da Tabela 1 mostram que o preparo da amostra onde se utilizou a homogeneização do mamão em processador pôde ser logo descartado. Neste tipo de preparo, onde a polpa/casca é transformada em uma pasta homogênea, não foi detectada a presença de BITC após nenhuma das extrações. Provavelmente, os vacúolos onde se encontra a mirosinase no interior da célula ${ }^{10,17}$ foram destruídos durante o processamento, diminuindo drasticamente a possibilidade de contato entre esta enzima e o substrato, evitando assim a formação de ITCs. O preparo da amostra onde os frutos são cortados em cubos mostrou bons resultados para extrações a temperatura ambiente. Neste tipo de preparo, a maior parte das células vegetais do mamão é preservada e a ação do equipamento usado na extração (Ultra-Turrax, ultrassom ou gral) faz com que o mecanismo de formação de ITCs entre em ação.

Em relação à extração com acetato de etila, o procedimento efetuado com solvente mantido a $4{ }^{\circ} \mathrm{C}$ mostrou resultados menos eficazes que com o uso de solvente a temperatura ambiente. Este resultado pode ser atribuído à maior dificuldade de solubilização dos isotiocianatos em solvente resfriado e/ou à desativação da mirosinase pela baixa temperatura.

As concentrações de isotiocianatos obtidas para amostras cortadas em cubos (amostra 1 - Tabela 1) mostraram que as extrações efetuadas, empregando o mesmo tempo (15 min), por ultrassom (US) e por maceração em gral têm resultados equivalentes. Na tentativa de melhorar o processo de extração de ITCs nesta matriz, aplicou-se a técnica de dispersão da matriz em fase sólida, DMFS - Matrix Solid Phase Dispersion - $M S P D,{ }^{30-32}$ efetuando outro ensaio com a adição de celite durante a extração no Ultra-Turrax. Este ensaio mostrou resultados ligeiramente superiores às extrações com ultrassom e no gral, mas mostrou-se menos eficiente que a extração sem celite. Nos três casos (US, gral e celite), como as concentrações de BITC foram inferiores aos da extração com Ultra-Turrax, estes procedimentos foram rejeitados. 


\section{Seletividade}

A seletividade de um método reside na capacidade de se identificar, de maneira inequívoca, o analito considerado. No caso específico deste estudo, a seletividade deste método foi avaliada através da injeção, nos dois sistemas - CG-DFC e CG-DNP - do extrato da amostra de mamão obtido de acordo com o procedimento experimental apresentado. Nos dois sistemas cromatográficos são obtidos cromatogramas "limpos" com apenas um pico cujo $t_{R}$ coincide com o do padrão de BITC. A Figura 1 mostra os cromatogramas que exibem apenas um pico dos extratos da amostra de mamão nos dois sistemas cromatográficos. A confirmação destes resultados, feita por espectrometria de massas (CG-DSM), assegurou a identidade do pico da amostra que tem o mesmo $t_{R}$ e espectro de massa que o padrão de BITC.
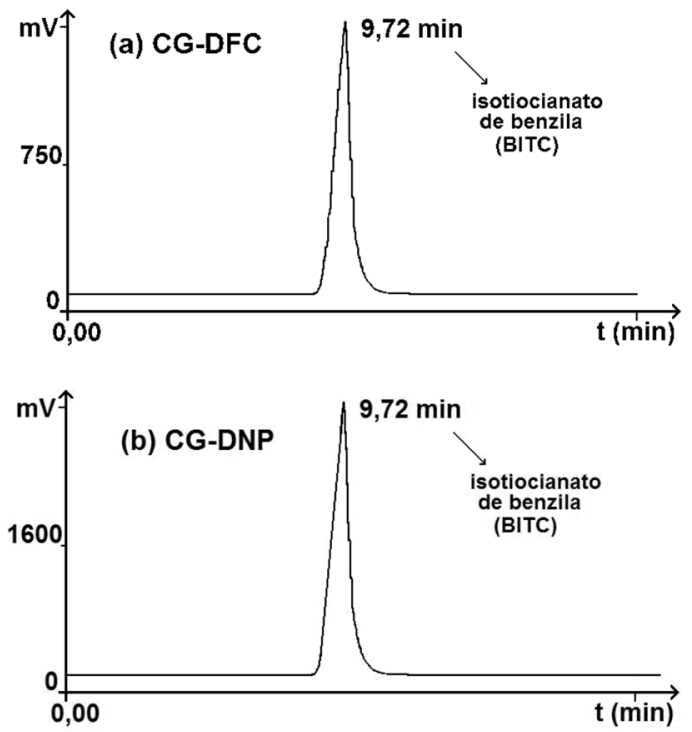

Figura 1. Cromatogramas do extrato da amostra de mamão nos sistemas (a) CG-DFC e (b) CG-DNP; coluna DB-1701 P (28,5 m x 0,32 mm x 0,25 $\mu \mathrm{m})$; programação de temperatura de 50 a $130{ }^{\circ} \mathrm{C}\left(25^{\circ} \mathrm{C} / \mathrm{min}\right)$ e de 130 a $200{ }^{\circ} \mathrm{C}$ $\left(5^{\circ} \mathrm{C} / \mathrm{min}\right)$; injetor splitless a $225^{\circ} \mathrm{C} ; V_{\text {injeção }} 0,8 \mu \mathrm{L}$, fluxo de gás de arraste (hélio) de 1,1 $\mathrm{mL} / \mathrm{min}$

Cabe ressaltar o fato do isotiocianato de benzila ser uma substância de ocorrência natural no mamão e a extração com AcOEt deste fruto mostrará normalmente a presença de BITC. Assim, torna-se impossível a análise de uma "amostra em branco" (sem o analito) e a obtenção de um cromatograma "limpo", sem picos. A análise por CG-DFC ou -DNP de seus extratos não produz cromatogramas sem picos, uma vez que não existe uma matriz isenta deste analito. Estes ensaios comprovaram que não existem, nesta matriz, interferentes que contenham em sua estrutura $\mathrm{S}, \mathrm{N}$ ou $\mathrm{P}$.

\section{Linearidade e faixa de trabalho para o sistema $C G-D F C$}

Através da aplicação das soluções padrão de BITC no sistema CG-DFC (seletivo para o enxofre) obteve-se os resultados da Tabela 2 e foi possível compor a curva de calibração com sete pontos exposta na Figura 2. Inicialmente foi usado um intervalo com concentrações de isotiocianato situadas na faixa de 5 a $56 \mu \mathrm{g} / \mathrm{mL}$.

Para se avaliar a homogeneidade na variância dos resíduos foi usado o teste de Cochran, que foi aplicado para o conjunto total dos sete pontos da curva e somente para os cinco pontos de maior concentração (série 2 da Figura 2). Nos dois casos o resultado do teste indicou que as variâncias são homogêneas para os intervalos usados, ou seja, há homocedasticidade nas duas faixas de trabalho consideradas.

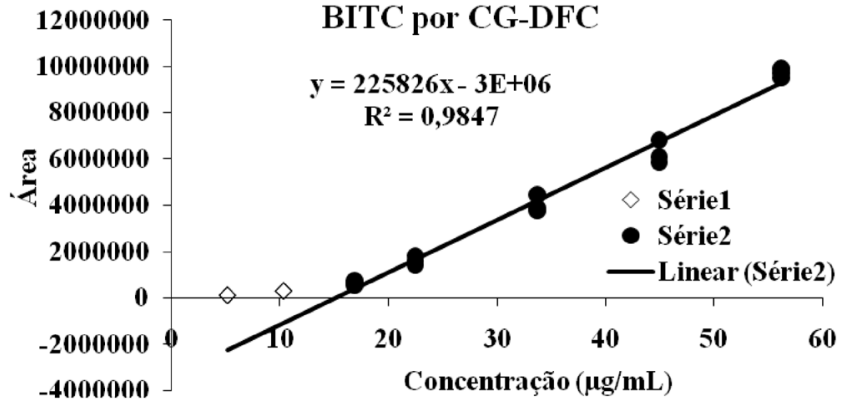

Figura 2. Curva padrão de BITC obtida no sistema $C G-D F C(n=3)$ utilizando as condições de análise descritas na Parte Experimental

Tabela 2. Resultados das análises das soluções padrão de BITC no sistema CG-DFC efetuadas em triplicata; coluna DB-1701 P (28,5 m x $0,32 \mathrm{~mm} \times 0,25 \mu \mathrm{m})$ da $\mathrm{J} \& \mathrm{~W}$ Scientific, programação de temperatura de 50 a $130{ }^{\circ} \mathrm{C}\left(25^{\circ} \mathrm{C} / \mathrm{min}\right)$ e de 130 a $200{ }^{\circ} \mathrm{C}\left(5^{\circ} \mathrm{C} / \mathrm{min}\right)$, injetor splitless a $225^{\circ} \mathrm{C}$ (tempo de abertura da válvula de $1 \mathrm{~min}$ ); volume de injeção de $0,8 \mu \mathrm{L}$ e fluxo de gás de arraste (hélio) de $1,1 \mathrm{~mL} / \mathrm{min}$

\begin{tabular}{lcc}
\hline padrão de BITC $(\mu \mathrm{g} / \mathrm{mL})$ & Média das áreas & $\begin{array}{c}\text { Coeficiente de } \\
\text { variação }\end{array}$ \\
\hline 5,18 & 99549 & 1,27 \\
10,36 & 283022 & 2,08 \\
16,86 & 642222 & 15,36 \\
22,48 & 1617133 & 13,09 \\
33,72 & 4024184 & 9,04 \\
44,96 & 6243092 & 7,87 \\
56,20 & 9686286 & 2,18 \\
\hline
\end{tabular}

A inspeção visual da curva da Figura 2 mostra que para os dois pontos de menor concentração (série 1 da Figura 2), a resposta do detector é diferente da obtida para os pontos mais concentrados (série 2 da Figura 2). Isto sugere que o fator de resposta do DFC, nas faixas de baixa e alta concentração do analito, é diferente. Isto se reflete no coeficiente de correlação $\left(\mathrm{R}^{2}\right)$ calculado nos dois intervalos de trabalho. O $\mathrm{R}^{2}$ para o intervalo contendo os sete pontos da curva foi de 0,9546 , enquanto que para a faixa de trabalho com cinco pontos (serie 2 da Figura 1), o R² é 0,9847. O coeficiente de correlação linear (r), calculado para o conjunto com sete pontos foi de 0,9770 , e para o conjunto de cinco pontos foi de 0,9923 .

Os valores encontrados para $\mathrm{r}$ e $\mathrm{R}^{2}$ calculados para os dois conjuntos de sete e de cinco pontos conduziram ao estabelecimento de uma faixa de trabalho mais adequada, como sendo no intervalo de 16,86 a $56,2 \mu \mathrm{g}$ BITC/mL, por ter apresentado um coeficiente de correlação superior e o coeficiente de correlação linear mais próximo da unidade. No entanto, este resultado não pode ser usado isoladamente para se testar a linearidade. A significância estatística da curva é feita pelo teste F. Para isso foi utilizada a análise de variância da regressão (ANOVA) para se confirmar a linearidade da curva. Para esta curva, o valor de $\mathrm{F}_{\text {calculado }}(=834,8)>\mathrm{F}_{\text {de significacão }}\left(=3,5 \times 10^{-13}\right)$, o que demonstra a significância da regressão e ratifica a linearidade da faixa de trabalho estudada. $\mathrm{O}$ valor calculado da probabilidade de hipótese nula $(\mathrm{P})$, calculado também através da ANOVA, foi $3,048 \times 10^{-08}$, indicando que a reta não passa pela origem, o que é facilmente visualizado na Figura 2.

Os limites de detecção (LD) e de quantificação (LQ) foram calculados utilizando dados da regressão da curva de calibração. A equação da reta nesta faixa de trabalho é y $=255826 x-3 \cdot 10^{6}$. O 
cálculo destes limites forneceu os valores de $\mathrm{LD}=5,78 \mu \mathrm{g} / \mathrm{mL}$ e LQ $=19,29 \mu \mathrm{g} / \mathrm{mL}$.

\section{Linearidade e faixa de trabalho para o sistema $C G-D N P$}

Através da aplicação das soluções padrão de BITC no sistema CG-DNP (seletivo para nitrogênio) foi possível compor a curva de calibração exposta na Figura 3. Inicialmente foi usado um intervalo com concentrações de isotiocianato situadas na faixa de 0,10 a 50,15 $\mu \mathrm{g} / \mathrm{mL}$

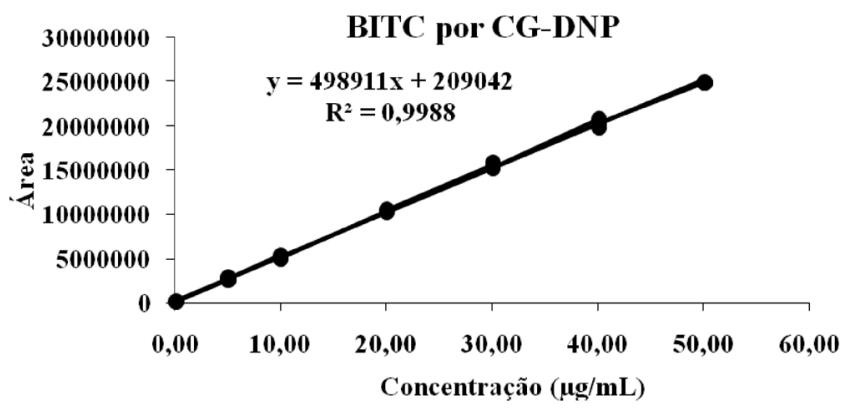

Figura 3. Curva padrão de BITC obtida no sistema $C G-D N P(n=3)$ utilizando as condições de análise descritas na Parte Experimental

A avaliação da homo/heterocedasticidade, na faixa de trabalho considerada para o CG-DNP, é feita pelo teste de Cochran. Este teste indicou que as variâncias são homogêneas neste intervalo, isto é, há homocedasticidade, e o método dos mínimos quadrados pode ser usado para a regressão linear da curva. A regressão da reta e a análise de variância ANOVA foram realizadas no Excel (MS Office) utilizando os resultados mostrados na Tabela 3.

O coeficiente de correlação da curva $\left(\mathrm{R}^{2}\right)$ obtido foi de 0,9988 e é considerado bem satisfatório. O coeficiente de correlação linear (r) foi de 0,9994, bem próximo da unidade, e expressa a relação de linearidade entre a concentração e a área na curva. A análise por ANOVA mostra um valor de $\mathrm{F}_{\text {calculado }}(=16088)$ superior ao $\mathrm{F}_{\text {de significação }}$ $\left(=2,74.10^{-29}\right)$ comprovando a linearidade do intervalo considerado neste estudo. $\mathrm{O}$ parâmetro $\mathrm{P}$ (probabilidade de hipótese nula) que deve
Tabela 3. Resultados das análises em triplicata das soluções padrão de BITC no sistema CG-DNP efetuadas em triplicata; coluna DB-1701 $\mathrm{P}(28,5 \mathrm{~m} \times 0,32 \mathrm{~mm} \times 0,25 \mu \mathrm{m})$ da $\mathrm{J} \& \mathrm{~W}$ Scientific, programação de temperatura de 50 a $130{ }^{\circ} \mathrm{C}\left(25^{\circ} \mathrm{C} / \mathrm{min}\right)$ e de 130 a $200{ }^{\circ} \mathrm{C}\left(5^{\circ} \mathrm{C} /\right.$ min), injetor splitless a $225{ }^{\circ} \mathrm{C}$ (tempo de abertura da válvula de 1 min); volume de injeção de $0,8 \mu \mathrm{L}$ e fluxo de gás de arraste (hélio) de $1,1 \mathrm{~mL} / \mathrm{min}$

\begin{tabular}{lcc}
\hline padrão de BITC $(\mu \mathrm{g} / \mathrm{mL})$ & Média das áreas & $\begin{array}{c}\text { Coeficiente de } \\
\text { variação }\end{array}$ \\
\hline 0,10 & 88826 & 9,72 \\
5,02 & 2694650 & 6,95 \\
10,03 & 5127661 & 4,09 \\
20,06 & 10376287 & 1,42 \\
30,09 & 15583937 & 2,12 \\
40,12 & 20298583 & 2,52 \\
50,15 & 24908963 & 0,20 \\
\hline
\end{tabular}

ser maior ou igual a 0,05 para a interseção, foi de 0,075 , portanto, pode-se considerar que a reta passa pela origem e que o método não está sujeito a erros sistemáticos.

O cálculo dos limites de detecção e de quantificação, usando o detetor DNP, foi feito usando a relação sinal/ruído (3:1 para o LD e 10:1 para o LQ). Para se estabelecer estes limites foi usada a menor concentração de BITC capaz de fornecer um pico que tenha três ou dez vezes a amplitude do sinal de ruído. Os ensaios foram feitos em triplicata para cada concentração testada. Os valores dos limites calculados a partir destes ensaios foram $\mathrm{LD}=0,038 \mu \mathrm{g} / \mathrm{mL}$ e $\mathrm{LQ}=$ $0,100 \mu \mathrm{g} / \mathrm{mL}$. O Quadro 1 resume todos os parâmetros de validação do método de análise de BITC pelos sistemas CG-DFC e CG-DNP citados neste estudo.

Taxa de recuperação

Outro parâmetro importante na validação de um método é a percentagem de recuperação. A taxa de recuperação de um analito é a razão entre a quantidade do analito adicionado à amostra para ser quantificado por

Quadro 1. Resumo dos parâmetros de validação usados na validação do método de análise de isotiocianato de benzila por CG-DFC e por CG-DNP

Análise de isotiocianato de benzila

Parâmetros de validação

Faixa de trabalho sistema CG-DFC

sistema CG-DNP

16,86 a $56,2 \mu \mathrm{g}$ BITC/mL

0,10 a $50,15 \mu \mathrm{g}$ BITC/mL

Teste de Cochran (homogeneidade das variâncias)

Regressão linear

Coeficiente angular

Coeficiente linear

Coeficiente de correlação linear

$\mathrm{C}_{\text {calc }}=0,510<\mathrm{C}_{\text {tab }}=0,684$ homocedástico

$$
\begin{gathered}
\mathrm{Y}=255826 \mathrm{x}-3.10^{6} \\
\mathrm{~b}=255826 \mathrm{~mL} / \mu \mathrm{g}
\end{gathered}
$$$$
\mathrm{a}=3.10^{6}
$$$$
\mathrm{r}=0,9923
$$$$
\mathrm{R}^{2}=0,9847
$$

Coeficiente de correlação

significância da regressão:

$\mathrm{F}_{\text {calculado }}>\mathrm{F}_{\text {de significação }}$

$$
\mathrm{F}_{\text {calc }}=834,8285>\mathrm{F}_{\text {sig }}=3,51 \text {. }
$$

$10^{-13}$

linearidade da faixa de trabalho

Limite de detecção

$\mathrm{LD}=5,78 \mu \mathrm{g} / \mathrm{mL}$

$\mathrm{LQ}=19,29 \mu \mathrm{g} / \mathrm{mL}$
$\mathrm{C}_{\text {calc }}=0,551<\mathrm{C}_{\mathrm{tab}}=0,561$ homocedástico

$$
\begin{gathered}
\mathrm{Y}=498911 \mathrm{x}+209042 \\
\mathrm{~b}=498911 \mathrm{~mL} / \mu \mathrm{g} \\
\mathrm{a}=209042 \\
\mathrm{r}=0,9994 \\
\mathrm{R}^{2}=0,9988 \\
\mathrm{~F}_{\text {calc }}=16088,185>\mathrm{F}_{\mathrm{sig}}=2,75 .
\end{gathered}
$$$$
10^{-29}
$$

linearidade da faixa de trabalho

$$
\mathrm{LD}=0,038 \mu \mathrm{g} / \mathrm{mL}
$$

$\mathrm{LQ}=0,100 \mu \mathrm{g} / \mathrm{mL}$ 
um método analítico e a quantidade teórica adicionada à matriz antes do procedimento. No caso do isotiocianato de benzila, que é uma substância de ocorrência natural do mamão, o ensaio de recuperação teve que considerar a quantidade original de BITC presente na amostra (valor base) visto que esta substância é inerente à matriz. Assim, em paralelo a cada ensaio de recuperação, foram feitas 10 determinações da concentração de isotiocianato da amostra em estudo. Para o cálculo da \% de recuperação de isotiocianato, o valor da concentração original de BITC na amostra - valor base - foi somado à concentração teórica deste analito que foi adicionada durante cada ensaio de recuperação. Os níveis de fortificação foram estabelecidos considerando a soma do valor base de BITC e da concentração que foi adicionada à matriz. Os ensaios foram feitos em três níveis de fortificação e os resultados da recuperação de BITC estão na Tabela 4. Para os três níveis de fortificação, 1,40; 2,80 e 4,15 $\mu \mathrm{g} / \mathrm{mL}$, a média das recuperações foi $90,64 \%$. Considerando a repetitividade do método como o coeficiente de variação (CV), a partir da Tabela 4, pôde-se calcular o CV como sendo 2,88\%. Estes resultados são coerentes com a faixa de recuperação aceitável de 70 a $110 \%$ recomendada pelo Codex Alimentarius $^{33}$ e também pela União Européia ${ }^{27,28}$.

Tabela 4. Taxas de recuperação de BITC em mamão e coeficiente de variação do método

\begin{tabular}{lcc}
\hline nível de fortificação & $\begin{array}{c}\text { faixa de recuperação } \\
(\%)\end{array}$ & $\begin{array}{c}\text { Média } \\
(\%)\end{array}$ \\
\hline $1,40 \mu \mathrm{g} / \mathrm{mL}$ & 92,06 a 93,76 & 92,80 \\
$2,80 \mu \mathrm{g} / \mathrm{mL}$ & 86,59 a 88,24 & 87,51 \\
$4,15 \mu \mathrm{g} / \mathrm{mL}$ & 90,34 a 93,50 & 91,61 \\
Média da recuperação & 90,64 & \\
\%CV (repetitividade) & 2,88 & \\
\hline
\end{tabular}

\section{Aplicação do método validado}

Determinações de isotiocianato de benzila, seguindo o método validado descrito na Parte Experimental, foram efetuadas em 78 amostras de mamão. Os frutos analisados de Carica papaya eram provenientes de cultivo orgânico e convencional e boa parte pertence à variedade Formosa, que é amplamente consumida pela população brasileira. Cada fruto foi enquadrado na escala de maturação proposta por Balbino ${ }^{26} \mathrm{e}$ todos os resultados obtidos estão dispostos nas Tabelas 5a e 5b. Estas análises permitiram a determinação da concentração média de 16,23 $\mu \mathrm{g}$ de BITC/g de amostra de mamão.

Segundo dados da literatura, a concentração de isotiocianato nos frutos diminui com a sua maturação ${ }^{1,3,29}$. Os resultados mostram uma tendência de se encontrar quantidades maiores de BITC quanto menor for a maturação, mas isto não é tão evidente em todos os conjuntos de amostras. Existem mesmo diferenças razoáveis entre os resultados de BITC obtidos para amostras de mesmo grau de maturação. Deve ser levado em consideração que as amostras analisadas provêm de cultivos e de áreas diferentes, o que pode gerar variações na composição do fruto. Esta diferença poderia ser atribuída à variabilidade entre as plantas/frutos, à sazonalidade, à sua origem e à forma de cultivo. Foram feitos os cálculos das médias de BITC das variedades Papaia e Formosa cultivadas de maneira orgânica e convencional (Tabela 6).

Considerando o total das amostras analisadas, os resultados obtidos mostram que os frutos da variedade Papaia apresentaram concentrações de BITC superiores aos da variedade Formosa. Além disso, comparando os resultados obtidos para as amostras orgânicas e convencionais, constatou-se que os frutos orgânicos mostraram concentrações de isotiocianatos maiores que os convencionais. No entanto, para se confirmar este tipo de resultado, seria necessário realizar estas análises com um número bem maior de amostras.
Tabela 5a. Resultados das análises de BITC em amostras de mamão papaia $(\mathrm{P})$ e também da variedade Formosa $(\mathrm{F})$ provenientes de cultivo convencional. Cada fruto coletado representa uma amostra, e cada resultado representa a média de duas replicatas da mesma amostra

\begin{tabular}{lccccc}
\hline \multicolumn{5}{c}{ Cultivo convencional } & \\
Amostra & BITC & Amostra & $\begin{array}{c}\text { BITC } \\
(\mu \mathrm{g} / \mathrm{g})\end{array}$ & Amostra & $\begin{array}{c}\text { BITC } \\
(\mu \mathrm{g} / \mathrm{g})\end{array}$ \\
\hline F 1 & 19,22 & F 23 & 14,15 & P 3 & 7,66 \\
F 2 & 19,24 & F 24 & 17,33 & P 4 & 7,67 \\
F 3 & 14,04 & F 25 & 17,10 & P 5 & 7,05 \\
F 4 & 14,12 & F 26 & 17,91 & P 6 & 7,02 \\
F 5 & 20,00 & F 27 & 16,94 & P 7 & 7,65 \\
F 6 & 19,80 & F 28 & 5,74 & P 8 & 7,60 \\
F 7 & 19,13 & F 29 & 6,32 & P 9 & 7,32 \\
F 8 & 19,09 & F 30 & 6,35 & P 10 & 7,28 \\
F 9 & 18,85 & F 31 & 3,99 & P 11 & 3,80 \\
F 10 & 18,89 & F 32 & 3,96 & P 12 & 3,81 \\
F 11 & 18,80 & F 33 & 17,10 & P 13 & 21,89 \\
F 12 & 18,82 & F 34 & 17,10 & P 14 & 21,90 \\
F 13 & 16,75 & F 35 & 16,95 & P 15 & 26,92 \\
F 14 & 16,78 & F 36 & 16,93 & P 16 & 25,89 \\
F 15 & 6,92 & F 37 & 17,33 & P 17 & 47,10 \\
F 16 & 6,91 & F 38 & 17,32 & P 18 & 47,2 \\
F 17 & 18,87 & F 39 & 15,30 & P 19 & 9,27 \\
F 18 & 19,11 & F 40 & 15,33 & P 20 & 9,30 \\
F 19 & 20,31 & F 41 & 16,13 & P 21 & 22,18 \\
F 20 & 25,18 & F 42 & 16,17 & P 22 & 22,17 \\
F 21 & 26,00 & P 1 & 7,30 & & \\
F 22 & 14,15 & P 2 & 7,27 & & \\
\hline & & & & &
\end{tabular}

Tabela 5b. Resultados das análises de BITC em amostras de mamão papaia $(\mathrm{P})$ e também da variedade Formosa $(\mathrm{F})$ provenientes de cultivo orgânico. Cada fruto coletado representa uma amostra, e cada resultado representa a média de duas replicatas da mesma amostra

\begin{tabular}{lccccc}
\hline \multicolumn{5}{c}{ Cultivo orgânico } \\
Amostra & $\begin{array}{c}\text { BITC } \\
(\mu \mathrm{g} / \mathrm{g})\end{array}$ & Amostra & $\begin{array}{c}\text { BITC } \\
(\mu \mathrm{g} / \mathrm{g})\end{array}$ & Amostra & $\begin{array}{c}\text { BITC } \\
(\mu \mathrm{g} / \mathrm{g})\end{array}$ \\
\hline F 43 & 19,20 & P 24 & 10,16 & P 29 & 25,30 \\
F 44 & 19,12 & P 25 & 47,29 & P 30 & 25,30 \\
F 45 & 4,75 & P 26 & 47,33 & P 31 & 7,84 \\
F 46 & 4,72 & P 27 & 17,44 & P 32 & 7,87 \\
P 23 & 10,13 & P 28 & 17,48 & & \\
\hline
\end{tabular}

Tabela 6. Médias ponderadas das concentrações de BITC as amostras de mamão Papaia e Formosa (Carica papaya e Carica papaya Var. Formosa, respectivamente) cultivados de forma convencional e orgânica

médias das concentrações de BITC $(\mu \mathrm{g} / \mathrm{g})$

\begin{tabular}{lcc} 
& convencional & orgânico \\
\hline Formosa & 15,87 & 11,95 \\
Papaia & 15,24 & 21,62 \\
média & & \\
ponderada total & 15,65 & 18,85 \\
\hline
\end{tabular}




\section{CONCLUSÃO}

O método proposto para análise de isotiocianatos empregando extração com acetato de etila e posterior análise por CG com detectores seletivos (DFC e DNP) demonstrou ser bastante adequado para a gama de amostras usada, além de ser de execução simples. O uso de detectores seletivos permitiu a obtenção de cromatogramas isentos de interferentes para o tipo de matriz, o que facilita muito a quantificação da substância de interesse. Observou-se comportamento linear da curva na faixa de concentração de BITC usualmente encontrada nos alimentos. Ficou evidente a maior sensibilidade e fator de resposta do detector de $\mathrm{N}$ e $\mathrm{P}$ frente ao analito que o detector DFC. A determinação de isotiocianato de benzila usando o DNP apresentou limites de detecção e quantificação menores, possibilitando a quantificação de concentrações baixas de BITC sem se observar efeito de matriz e utilizando uma faixa linear de trabalho mais ampla. As determinações de BITC em mamão forneceram um valor médio de concentração em todas as amostras analisadas de 16,23 $\mu \mathrm{g} / \mathrm{g}$. Constatou-se certa tendência de haver concentrações mais elevadas de isotiocianatos nas amostras de cultivo orgânico.

\section{REFERÊNCIAS}

1. Fahey, J. W.; Zalkmann, A. T.; Talalay, P.; Phytochemistry 2001, 56, 5.

2. Bones, A. M.; Rossiter, B.; Physiol. Plant. 1996, 97, 194.

3. Vermorel, M.; Heaney, R. K.; Fenwick, G. R.; J. Sci. Food Agric. 1988, 44, 321.

4. Mawson, R.; Heaney, R. K.; Piskula, M.; Kozlowska, H.; Die Nãhrung 1995, 39, 21

5. Musk, S. R. R.; Astley, S. B.; Stephenson, P.; Hubert, R. B.; Johnson, I. T.; Food Chem. Toxicol. 1995, 33, 31.

6. Wilson, R. K.; Kwan, T. K.; Kwan, C. Y.; Sorger, G. J.; Life Sci. 2002, $71,497$.

7. Adebiyi, A.; Adaikan, P. G.; Prasad, R. N. V.; Life Sci. 2003, 74, 581.

8. Wijnands, M. V. W.; Van Erk, M. J.; Doornbos, R. P.; Krul, C. A. M.; Woutersen, R. A.; Food Chem. Toxicol. 2004, 42, 1629.

9. Talalay, P.; Proceedings of the American Philosophical Society 1999, $143,52$.

10. Morant, A. V.; Jorgensen, K.; Jorgensen, C.; Paquette, S. M.; SánchezPerez, R.; Moller, B. L.; Bak, S.; Phytochemistry 2008, 69, 1795.

11. Tawfiq, N.; Heaney, R. K.; Plumb, I. A.; Fenwick, G. R.; Musk, S. R.; Williamson, G.; Carcinogenesis 1995, 16, 1191.
12. Fahey, J. W.; Zhang, Y.; Talalay, P.; Proc. Natl. Acad. Sci. U. S. A. 1997, 94, 10367

13. Gamet-Payrastre, L.; Li, P.; Lumeau, S.; Cassar, G.; Dupont, M. -A.; Chevolleau, S.; Gasc, N.; Tuljiez, J.; Terce, F.; Cancer Res. 2000, 60, 1426.

14. Nakamura, Y.; Morimitsu, Y.; Uzu, T.; Ohigashi, H.; Murakami, A.; Naito, Y.; Nakagawa, Y.; Osawa, T.; Uchida, K.; Cancer Lett. 2000, 157, 193.

15. Volden, J.; Borge, G. I.; Bengtsson, G. B.; Hansen, M.; Thygesen, I. E.; Wicklund, T.; Food Chem. 2008, 109, 595.

16. Shapiro, T. A.; Fahey, J. J. W.; Wade, K. L.; Stephenson, K. K.; Talalay, P.; Cancer Epidemiology, Biomarkers \& Prevention 2001, 10, 501.

17. Geshi, N.; Andreasson, E.; Meijer, J.; Rask, L.; Brandt, A.; Plant Physiol. Biochem. 1998, 36, 583.

18. Kuroiwa, Y.; Nishikawa, A.; Kitamura, Y.; Kanki, K.; Ishii, Y.; Umemura, T.; Hirose, M.; Cancer Lett. 2006, 241, 275.

19. Kermanshai, R.; McCarry, B. E.; Rosenfeld, J.; Summers, P. S.; Weretilnyk, E. A.; Sorger, G. J.; Phytochemistry 2001, 57, 427.

20. D'agostini, F.; Izzotti, A.; Balansky, R. M.; Bennicelli, C.; De Flora, S.; Mutat. Res. 2005, 591, 173.

21. Kuang, Y. -F.; Chen, Y. -H.; Food Chem. Toxicol. 2004, 42, 1711.

22. Callaway, E. C.; Zhang, Y.; Chew, W.; Chow, H. H. S.; Cancer Lett. 2004, 204, 23

23. Zhang, Y.; Yao, S.; Li, J.; Proc. Nutr. Soc. 2006, 65, 68.

24. Zhang, Y.; Li, J.; Tang, L.; Free Radical Biol. Med. 2005, 38, 70.

25. Vierheilig, H.; Bennett, R.; Kiddle, G.; Kaldorf, M.; Ludwig-Müller, J.; New Phytol. 2000, 146, 343.

26. Balbino, J. M. S.; Tese de Doutorado, Universidade Federal de Viçosa, Brasil, 1997.

27. http://ec.europa.eu/food/plant/resources/qualcontrol_en.pdf, acessada em Junho de 2008.

28. http://www.eurachem.org/guides/valid.pdf, acessada em Junho 2008.

29. Castro, I. M.; Anjos, M. R.; Silva, A. A.; Resumos da $28^{a}$ Reunião Anual da Sociedade Brasileira de Química, Poços de Caldas, Brasil, 2005.

30. Menezes Filho, A.; Navickiene, S.; Dórea, H. S.; J. Braz. Chem. Soc. 2006, 17, 874.

31. Cardoso, M. H. W. M.; Bastos, L. H. P.; Neves, T. S.; Abrantes, S.; Ciênc. Tecnol. Aliment. 2004, 24, 298.

32. Dórea, H. S.; Lima Sobrinho, L.; J. Braz. Chem. Soc. 2004, 15, 690.

33. Joint FAO/WHO Codex Alimentarius Commission; Methods of analysis and sampling. 2000, vol. 2A, part 1. 